\title{
Rich Interactions in Virtual Reality Exposure Therapy: A Pilot-Study evaluating a System for Presentation Training
}

\author{
Marius Koller \\ UniTyLab \\ Heilbronn University \\ Heilbronn, Germany \\ Philip Schfer \\ UniTyLab \\ Heilbronn University \\ Heilbronn, Germany
}

\author{
Daniel Lochner \\ UniTyLab \\ Heilbronn University \\ Heilbronn, Germany \\ Gerrit Meixner \\ UniTyLab \\ Heilbronn University
Heilbronn, Germany \\ Heilbronn University
Heilbronn, Germany
}

\begin{abstract}
Public Speaking Anxiety (PSA) or discomfort while speaking in public is a wide spread cognitive disorder. Exposure therapy offers the opportunity to treat patients suffering PSA by exposing them to the phobic stimulus. To plan and organize an in-vivo exposure takes a lot of effort in recruiting people for an audience and orchestrating their behavior. Virtual Reality (VR) offers the possibility to generate the audiences that can be controlled by an orchestrator according patient's individual needs. This paper explores a system that enables the therapists to richly interact verbally and non-verbally with immersed presenters. For evaluation, we conducted a study with 24 healthy participants in two groups (12 participants each). Our results indicate that the direct verbal interaction between an orchestrator outside the VR and an immersed presenter are enhancing the presenter's experience and increasing the efficiency of therapy process. The non-verbal dimension is realized that an orchestrator can takeover an avatar using a motion-tracking camera that controls then the avatar's movements. Our results indicate that the transition of animations and movements are not impacting the experience negatively.

Index Terms-Virtual Reality, Virtual Reality Exposure Therapy, Public Speaking Anxiety
\end{abstract}

\section{INTRODUCTION}

In this paper, we present a study that evaluates a system for the therapy of public speaking anxiety (PSA) allowing the therapist from outside the VR to richly interact with immersed patients by controlling an avatar in the audience (see figure 2). We expect that the concept of rich interactions is contributing to a more flexible and individual exposure therapy. In current practice of in-vivo exposure therapy, the therapist guides the patient through a phobic stimulus in a real world environment. In PSA this implies that an audience must be present. In exposure in-situ, therapists guide patient's imagination to visit or generated the phobic stimulus. VR opens the opportunity to deliver an individualized and fully controllable phobic stimulus using a therapeutic scenario.

Despite the expected improvement on exposure therapy by adding flexibility for therapists, this evaluation focuses on the patient's perception and experience of the live controlled avatar's interactions compared to standard pre-recorded animations. As stated in [1], [2], the development of mental health technology is done in two phases. Our system is currently in the first phase that demands initial evaluations to explore the possible usefulness in clinical settings. Therefore, we chose to conduct this pilot study to evaluate to what extend our design is influencing the participant's experience and presence.

Virtual Reality (VR) is applied in various domains and usecases like the training of workers in assembly tasks [3] or in safety behavior [4]. Further training applications can be found in medical environments for example in the training of minimal invasive interventions such as surgeries or dentist treatments [5].

Other applications implement systems for rehabilitation which focus for example on the rehabilitation of bodily functions, e.g. upper limb or after stroke events or on mental health. In the field of mental health, the possibility of treating anxiety disorders was researched first in the 1990ies with a concept for therapy of acrophobia [6]. With a 12-month prevalence of $18.1 \%$ [7] and a lifetime-prevalence of $28.8 \%$ [8], anxiety disorders are one of the most frequent mental disorders in the US and Europe.

Cognitive behavior therapy (CBT) offers with the exposure therapy a highly effective therapy method that has response rates that range between $80 \%-90 \%$ [8], [9]. In the course of the therapy, the patient's anxiety level is decreasing. Virtual Reality (VR) offers the possibility to generate the phobic stimulus in a virtual environment, called Virtual Reality Exposure Therapy (VRET). Various studies indicate the effectiveness [10], [11] of the therapy with response rates that are comparable to those of a in-vivo therapy [12]-[15], which is currently considered as the gold-standard. Furthermore, there is evidence that patients prefer VR compared to exposition in-vivo [16]

Most research on VRET focuses on the therapy method itself and its effectiveness compared to the traditional therapy which is conducted in-vivo or in-situ. With this work, we aim to enhance the therapist's possibility to perform the therapy by enriching a VRET-system. For this purpose, we developed a system that enables the therapist - who is orchestrating the VR 
while not immersed - to interact with the patient immersed in the VR by controlling a virtual character. The system aims to enable the therapist to provide rich and spontaneous verbal and non-verbal interactions which may offer a greater flexibility and higher degree of individualization in therapy. This concept is exemplified in the field of public speaking anxiety (PSA), in our case, we focus on fear of presentation.

The paper structures as follows: the next section explains the background of our research followed by related work, followed by a section that presents our system. The section after describes the methods we used and the study we conducted to answer our research question. Section VII presents our results followed by a discussion, limitations and conclusion. At the end of the paper, we present future work.

\section{BACKGROUND}

Public speaking anxiety is classified as a form of social anxiety disorder (SAD) by the American Association of Psychologists [17] and is listed as F40.1 in the ICD-10. There is evidence that it can be considered as a distinguished sub-type of SAD and has a prevalence of about $20 \%$ [18]. However, even more people feel uncomfortable when speaking in front of audiences, than are diagnosed with PSA. Both groups may experience negative impacts in their professional career and social life. Like other anxiety disorders, PSA can be treated using traditional exposure therapy or the more modern VRET. VRET-systems provide the patients a virtual scenario in which they get exposed to phobic stimuli, in our case the situation where the patients speak in public. In general, there are two common ways to generate the needed environment for VRET. First, the desired scenario can be set up in the real world and then be recorded using $360^{\circ}$ cameras. This allows a rather quick and cheap generation of an authentic high quality environment, but is fairly limited in terms of interaction. Second, scenarios can be computer generated virtual worlds which allows its designer to fully shape the phobic stimulus and all surroundings according to the therapeutic needs. However, these solutions are more expensive and time-consuming to develop.

Interactions between the presenter and an audience play an important role in the therapy of PSA. In this case, the interactions are performed using the verbal and non-verbal channel. Non-verbal interactions include the emotions of the audience, e.g. attentive, bored or even disturbing, or the gesticulations of virtual avatars. The emotion of an audience influences the speakers performance, i.e. a negative audience provokes a greater anxiety [19]. The verbal component of interactions may contain questions and comments towards the presenter and other distractions.

The concept of presence, which is defined as the "sense of being there" [20], [21], is one key factor in VRET. Research indicates that patient's presence is influencing the therapy outcome [11] and has a correlation to anxiety [22]. However, as Ling et al. conclude, the standard measures are representing the illusion of being involved in VR [22], the social component, which plays a role in PSA, is not covered with these standard measures [23]. Two more interrelated types of presence [24] were introduced: social presence and co-presence [25]. Co-presence is defined by [25] as subjective experience of being together with other humans while physically not co-located or being with computer-generated avatars. Social-presence relates to interactions which implies that a user feels the presence of another individual [25]. Both can describe the sense that we want to address with our system. To assess this, Poeschl and Doering propose a questionnaire that is covering these phenomenon [26].

Systems implement the social and interactive components of exposure therapy and in presentation training in various ways. The virtual characters are among the most central elements in a social VR experience. In modern VR systems the virtual characters are non-static and perform animations that can be implemented in various ways. The most common method is to manually animate the character by adjusting the movement of the body joints in a 3D character animation program, e.g. Autodesk Maya ${ }^{1}$. Another possibility is to pre-record movements of real humans using a motion capture system and assign them to the character. While the two previous approaches use pre-recorded animations, the third method would be to input the animations in real-time using a motion tracking camera that translates the movements of a human to the movement of a virtual character. This method offers the highest flexibility of a system as the user can react to every situation spontaneously.

\section{RELATED WORK}

With the advance in technology, the treatment of PSA or discomfort when speaking in public is researched using technology, e.g. the internet or VR. Conventionally, the training may take place using a real audience and a trainer that is giving feedback on the performance. Systems aim to imitate these two parts in different ways. RoboCOP is a fully automated presentation coach that uses a human-like robot which gives feedback using the robot as coach [27].

Virtual audiences play an important role in the delivery of training and therapy [28] as they are providing the (phobic) stimulus in these settings. In research there are studies that use neutral audiences [19] while others use expressive audiences [29], [30]. In therapy, virtual audiences provide the opportunity to conduct an effective therapy as the virtual audiences can be controlled easier and, therefore, enable the therapists to enhance the patient's quality of life.

In the research of Chollet et al. [29], the role of the a therapist - or rather trainer - was replaced by the audience as interface delivering feedback using different states of mood [29]. The behavior of the audience should in these cases represent or feedback the performance of the speaker. The results show that a system using an interactive audience can increase the engagement and improve the overall speaking performance. For an objective feedback generation, behavioral

\footnotetext{
${ }^{1}$ https://www.autodesk.com
} 
and performance data can, such as word-count or gaze direction, be collected [31].

Applications that offer training and practice for presentation skills in VR also made their way in Apple's AppStore and Google's PlayStore. These apps benefit from the performance modern smart phones offer. These applications (as for instance VirtualSpeech ${ }^{2}$ ) offer different environments with a static audience that is not reacting to a speaker's performance or questions.

Lindner et. al show in their study that off the shelf consumer hardware and software provide an effective therapy for public speaking anxiety [32]. They conducted a two-armed study comparing a traditional therapist-led therapy with a self-led exposure. After their therapy, both groups get a four-week period of in-vivo transition, which contains exercises that the participants are asked to perform in-vivo. After this, the participants showed a significant decrease in self-reported anxiety.

These systems have in common that they do not allow a direct interaction with the presenter and the virtual audience. Consequently, the systems allow no spontaneous interactions from the trainer or therapist, which may be a further stimulus in therapy or training. The animations in these systems are static, which means that the set of animations is limited and are repeated in a random order. Users aware of this, may see recurrent movement pattern which can cause a break in presence.

\section{PRoposed SySTEM}

In exposure therapy, a patient gets exposed to a phobic stimulus which a therapist needs to orchestrate and individualize [33]. Consequently, during the therapy of PSA, therapists need to be able to create individualized phobic stimuli, too. One possible stimulus might be the interaction with the virtual audience on both, the verbal and non-verbal channel.

VR-systems can use pre-defined animations for non-verbal and pre-recorded statements for the verbal interaction. Both can be triggered by the orchestrator ${ }^{3}$ when appropriate. In contrast, our system is designed to enable rich interactions between the orchestrator and the person immersed during a presentation. To do so, our system records the orchestrators upper-body movement and maps it to the chosen avatar's in real-time. The orchestrator chooses one avatar from the audience that is then controlled by the motion-tracking because we want to address the one-to-one interaction with our system. For motion-tracking we use the depth camera Microsoft Kinect v2. As depicted in Fig. 1, the orchestrator is situated outside of the VR and controlling the virtual audience. The presenter is immersed while he/she is holding the presentation. In our setting both users were physically located in two different rooms.

\footnotetext{
${ }^{2}$ https://virtualspeech.com/

${ }^{3}$ We use the term orchestrator instead of therapist as we did not involve therapists in the evaluation.
}

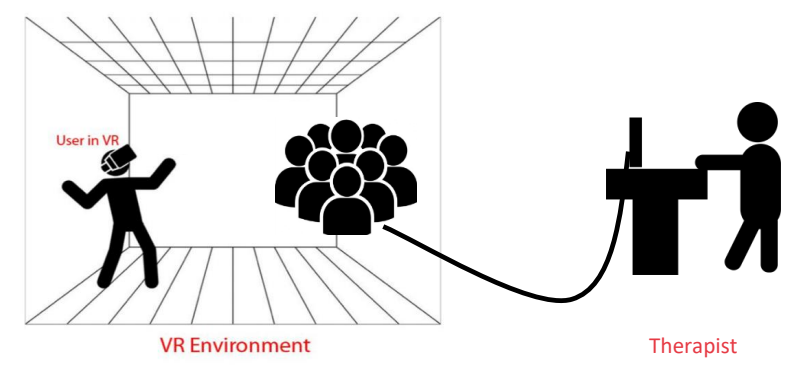

Fig. 1. Physical and virtual locations of the presenter and orchestrator.

Furthermore, our system allows voice input by the orchtestrator using a regular headset. The verbal input appears in VR as statement from the selected avatar. We did not integrate any voice modulation in this stage of development, therefore the presenter ${ }^{4}$ will hear the orchestrator's voice. The presenter is immersed into the virtual world using the HTC Vive along with the wireless transmission from TP-Cast.

To fully interact with the presenter, the orchestrator has to be placed in front of the Kinect sensor so that the upper body can be tracked. To select an avatar for control, we developed an interface (see fig. 2, top) that a user can control using the computer mouse. Avatars are represented as they are placed in $\mathrm{VR}$, so the orchestrator is choosing a seat. When an avatar is selected, we defined a gesture for activation of the live-input. This gesture is necessary to allow the orchestrator to preselect an avatar and interact with the presenter when appropriate.

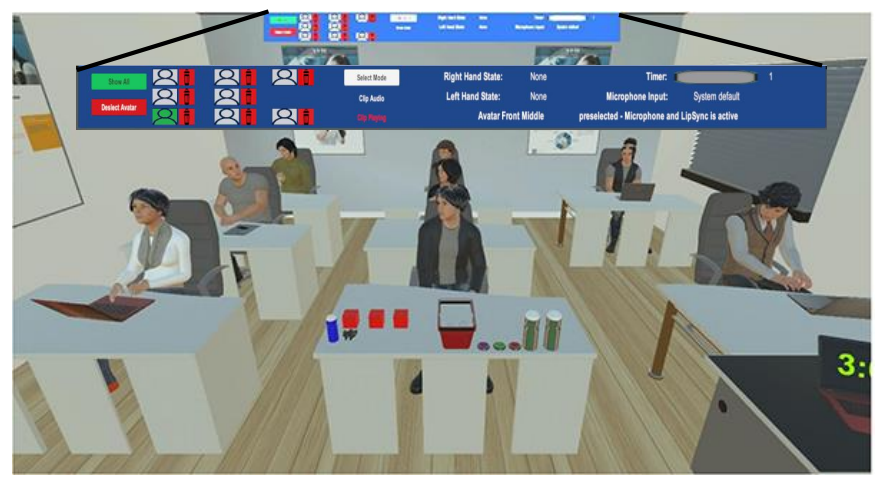

Fig. 2. The orchestrator's view with the administrative UI (top).

We tested different view angles for the orchestrator outside the VR, e.g. first-person view of the immersed presenter or from the back of the audience towards the presenter. We found it most convenient to mostly see the audience, as

\footnotetext{
${ }^{4}$ We use the term presenter instead of patient as we did evaluate the system with healthy participants.
} 
depicted in figure 2. This enables direct visual feedback on the behavior of avatars. We implemented an option to switch the view to the first-person perspective by pressing space on the keyboard that the orchestator is able to check the presenter's view.

The virtual environment was designed using the Unity ${ }^{5}$ editor. We oriented our design to small classrooms ubiquitous in university-settings. The room is equipped with ten tables and eight avatars. Three avatars have an open notebook in front of them. The avatars are displayed in a positive attitude towards the speaker (e.g. listening and nodding). The room has one window covered with a shade. The presentation is shown for the audience at the front wall. One table is standing between the starting-position of the presenters avatar and the audience. On this table, a notebook is placed which displays

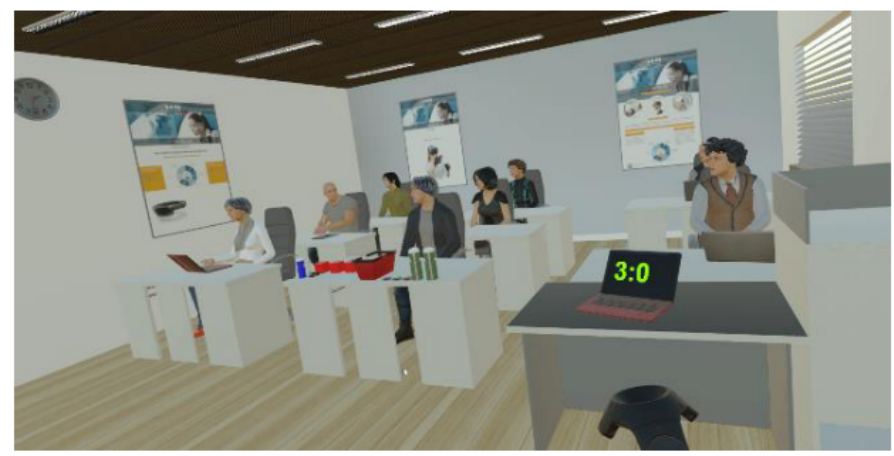

Fig. 3. View as seen by the presenter immersed in the VR.

a countdown of three minutes what should ensure that the presentations are kept within a time-frame and are comparable within our study setting.

The presenter immersed in the VR has no virtual representation of his/her own body (cf. fig. 3. For interaction with the slides and presentation we integrated a regular presenter that contains a laser pointer. The presenter was handled by the controller of the HTC Vive system. The participants were able to freely walk within the borders of the tracked room which has the dimensions of six by six meters.

\section{Methods}

We hypothesize that using our system, the immersed participants (i.e. the presenter and in future uses the patient) experience a more natural verbal interaction compared to prerecorded statements. Furthermore, we intend to evaluate the animation when an avatar is requesting to speak (i.e. rising the hand before asking a question to the presenter or answering a question from the presenter).

Our second hypothesis is that the transition from a synthetic animation to a human-controlled animation is not affecting the participant's experience. The system allows it to map orchestrator's movement to avatars in real time. The orchestrator is taking over the avatars only in cases when direct interaction is

\footnotetext{
${ }^{5}$ https://unity3d.com
}

needed. This means that immersed participant is experiencing regular animations most of the time.

To assess the hypothesis conducted a study that consisted of two groups:

- Experimental group: This group will experience the input from the orchestrator (voice and movement).

- Control group: This group will experience the regular animations that come with the avatar and the pre-recorded voice-input.

\section{A. Participants}

We recruited in total $\mathrm{N}=24$ participants ( 8 female, 16 male), all volunteered for the experiments without reward. If one of the following characteristics is true, we asked them to not participate for safety reasons: any form of epilepsy, any form of heart disease, disturbance of equilibrium, any form of mental disease, any form of a severe physical disease or pregnancy. To assess the parameters, we handed the participants the list of conditions and if one is present, they should just check a box I will not participate.

Participants were randomized and equally assigned to the experimental or control group. We used pseudo randomization that generated numbers that were randomly assigned to one of the groups. We printed the unique numbers that were folded and placed in a box. Each participant was asked to draw one of them, the number assigned them to the groups and was used as ID.

\section{STUdy DESIGN}

Participants have the role as presenter in front of the audience. At specified moments, they should interact with the audience. We defined two types of interactions: (1) presenter asking predefined questions; (2) an avatar is asking a question.

We prepared the presentation and questions for two reasons. First, participants may forget or may not want to ask questions during the presentation. Second, in the control condition we had to pre-record the avatar's answers. In order to respond properly to the questions, we had to know them in advance. Further answers to possible follow-up questions were not recorded.

\section{A. Procedure}

The study began for the participants with the completion of a consent form followed by a demographics questionnaire. With the 3-item self-rated Mini-SPIN questionnaire we could identify with an accuracy (efficiency) of $90 \%$ subjects with tendencies of general social anxiety disorders [34], [35]. As suggested from Wiltink at al. we cut-off subjects with ratings higher as 6 from our experiment for ethical reasons [35]. Then, we gave the participants a presentation about Italy that consisted of 8 slides. We used the cover-story that the participant is new in class and as a first get-together, all had to prepare a short presentation about one country, the participants chose Italy. The presentation included several facts about Italy (e.g. location in Europe and population) that every presenter could easily read from the slides projected to the wall in the 
VR. Each participant did go trough the slides and was granted the time needed till he or she felt comfortable to start the presentation.

After completion of the pre-experimental questions, we introduced the participants to the system. We asked them to wear the Head Mounted Display (HMD) and explained the relevant components for the experiment (e.g. the countdown of three minutes). We lead them towards a virtual wall until the HTC Vives chaperone-system was appearing and explained them the purpose of it mentioning that the participants are free to walk within these boundaries. As last part of the introduction we explained the controller, used as presenter in the scenario. When the participants completed the introduction, we were open for further questions. The participants had time to make themselves familiar with the VR and interaction until they felt ready to begin with the experiment.

The experiment started when the participants gave us a sign with an audio signal and start of the 3-minutes countdown. The interactions took place in the course of the presentation by questions from the presenter and virtual audience. To ensure that the presenter is asking them, we created a slide for each that he/she can simply read them. Both questions were answered by one avatar in the audience.

After the experiment, participants filled two questionnaires, one measuring the co-presence and social presence, the other measuring motion sickness. The study was concluded with a semi-structured interview gathering data on the presentation itself and the experience of the participants.

\section{B. Data Collection}

All participants were anonymized for the data-collection; each participant was assigned a random number that did not correspond with the order of participation. The informed consent and the other documents (e.g. questionnaire) were filed separately.

During the experiment, one researcher was observing the participant and taking paper notes. We told the participants that the observer is there for participants safety and will intervene in case of emergency, e.g. when the participants are in risk to walk into a physical object. We started our observation during the familiarization with the system, which included the first interaction along with a researcher. During the experiment, we primarily focused on the participant movement and interactions of any kind (e.g. interactions using the controllers or gesticulations). After the presentation, we noted any additional comments the participant made verbally before the interview started and, therefore, were not recorded.

During the interview, we asked question regarding the experience in VR and perception of the avatars and their interaction. We asked the participants to rate specific aspects (e.g. naturalness of animations) of their experience using a scale from 1 to 10, where 1 is worst and 10 is best. All interviews were audio-recorded for transcription. The transcriptions were used afterwards for further analysis as described in the next section.

\section{Data Analysis}

To analyze our transcribed interviews, we created a coding structure based on system features and experiences we expected the participants will have during the experiment. For example, we knew that our audience will interact verbally and non-verbally with the participants, so, we created a code for both dimensions of interaction. The codes are organized in a tree-structure that contains 29 codes in total. Figure 4 depicts an excerpt of the structure containing the part related to the VR. The branches not depicted in figure 4 due to space-restrictions, contain codes for the physical reactions (e.g. physical movement) of the participants during the experiment and possible enhancements in the participant's capability of presentations. The coding for the VR-part contains three dimensions:

- Interaction: Interaction with the audience in a verbal and non-verbal way and the interaction using the controller

- Emotion: This covers all emotional and subjective experiences of the participants during the experiment such as the general experience or feeling while presenting in VR.

- Virtual Environment: All aspects and contents of the virtual world such as room design and the aspects of the avatars.

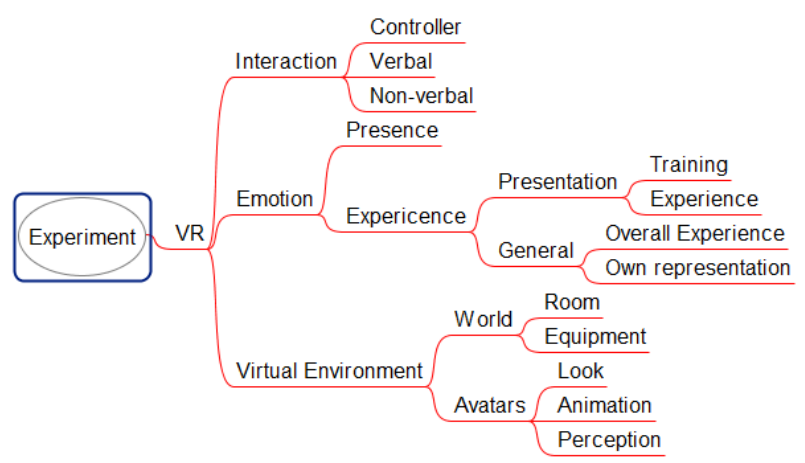

Fig. 4. The part of the coding structure representing the VR; not included are the codes that focus on the physical world.

We went through each transcription of interviews and analyzed all statements made during the interview and mapped the statements to our codes. Statements made after each question contained meta-information characterizing particular points of interest represented in the coding structure. Statements may also be related to multiple codes in our structure due to the complexity of human speech. For example the statement "the lady on the left raised her hand and wanted to ask a question" contains three information elements: first, the participant considers the virtual human as lady second, the participant perceived the animation as hand raising and, third, as a request to ask a question.

We analyzed the outcomes of the questionnaires and the ratings using statistical methods. As a first step, we analyzed 
whether our groups contain a significant difference in age, gender or previous experience in VR. Furthermore, we analyzed the social and co-presence questionnaire that should deliver us a level of presence in combination with our observations and the subjective ratings from the interviews.

\section{RESULTS}

As shown in table 1, we recruited in total 24 participants, 12 in each group. In total, we had 8 female and 16 male participants. Each group contained 4 female and 8 male participants. Overall, the participants had a medium age of 34.33 $(\mathrm{SD}=14.22)$. Participants in the experimental group had a mean of 30.83 ( $\mathrm{SD}=13.27)$, the mean in the control group was 37.83 ( $\mathrm{SD}=14.83$ ). Testing with the Mann-Whitney-U-Test, the null-hypothesis (distribution of the age between the groups is identical) has to be accepted ( $\mathrm{p}=0.219) .13$ participants $(54 \%)$ had already experiences in VR, these varied from brief handson up to longer gaming experiences. According to Fisher's exact test, the difference is not significant between the groups $(\mathrm{p}=0.100)$.

TABLE I

DISTRIBUTION OF PARTICIPANTS.

\begin{tabular}{|c|c|c|}
\hline & $\begin{array}{c}\text { Experimental Group } \\
\mathbf{N = 1 2}\end{array}$ & $\begin{array}{c}\text { Control Group } \\
\mathbf{N = 1 2}\end{array}$ \\
\hline Female & 4 & 4 \\
\hline Male & 8 & 8 \\
\hline Age & $\mathrm{M}=30.83 ; \mathrm{SD}=13.27$ & $\mathrm{M}=37.83 ; \mathrm{SD}=14.83$ \\
\hline Prev. experience with VR & 9 & 4 \\
\hline
\end{tabular}

The analysis of the scores on the Mini-SPIN, which is done by simply adding the chosen answer-items (between 0 and 4) [34], shows relatively low scores (see fig. 5. According to [35], all scores over 6 are indicating a tendency towards social phobias. In both groups the median is under this score. The experimental group shows a median of 3.5 which is higher than the median of the control group with 2.5. In both groups we find outliers with high scores, the highest score we received in the experimental group with 9 . We did not find a significant difference between the two groups $(\mathbf{T}=-1.83, d f=22, p=0.080)$.

The results of the social- and co-presence questionnaire which both groups filled after the experiment did not provide significant differences between the groups in general. The questionnaire item assessing the presenters reactions to the behavior of the audience shows a significant difference $(\mathbf{T}=-$ 2.695, $d f=22, p=0.014)$. The experimental has here a higher mean rating.

In both groups, we strove to assess the presence by observing the physical behavior and by coding statements from the interviews. During the experiment, we observed participants pointing towards the avatar who raised the hand. One participant even walked in the direction of an avatar to insist on closing the notebook in front. Furthermore, we observed gesticulation that indicated that the participants are behaving and feeling as in a real presentation.

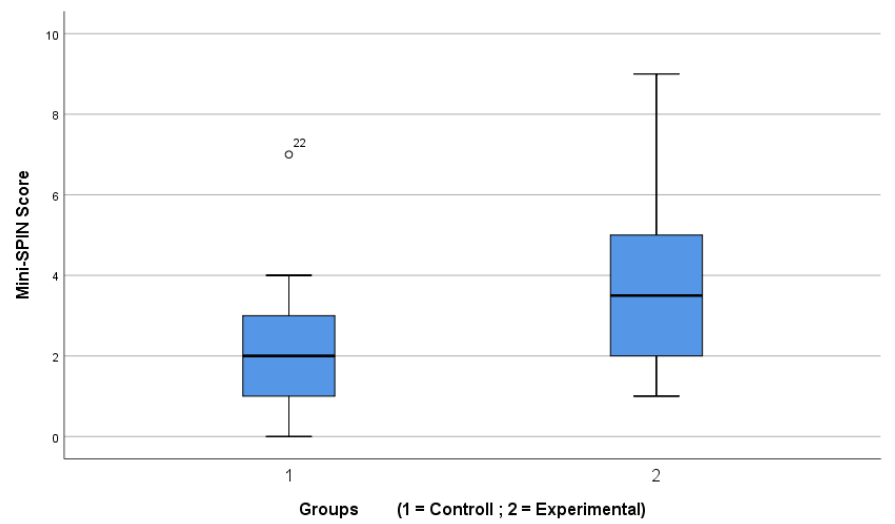

Fig. 5. Box-plot of scores of the Mini-SPIN sorted by the control and experimental group.

The interview data pointed towards a high presence, too. Most of the participants responded that they forgot about the physical environment and that an observer was in the same room. Further indications were the responses we got on the experience during the presentation. Participants stated that they perceived the presentation "like in the real world" along with the comment that they think the system can be used to exercise presentations.

As further indication of presence, we saw that participants in both groups related to virtual characters as human beings (coding: perception of avatars). $75 \%$ of the participants referred to them during the interviews as people, persons, classmates or audience. In six interviews (two in the control, four in the experimental group) we found more artificial characterizations. Three participants called them virtual people or humans, two acknowledged them as no real people. In one case, the participant called them with the technical term avatars.

In the experiment, we did not include the presenter's own virtual body representation. This implies that the presenters cannot see a body or extremities when looking towards the floor which might have a influence on presence [36]. In the interview we dedicated a question to this circumstance, asking if it irritated or affected the participants, which is represented as "own representation" in the coding structure. The majority $(66 \%)$ of them were not affected or irritated by the absence of their virtual body representation. One response to it was "When I am presenting I am not looking on my shoes, so I did not notice the absence". Three participants were undecided if it affected them. One for instance stated "One is used to see the feet. But knowing that I am in a virtual reality, it does not bother me in the experience.".

Five participants (three in the control, two in the experimental group) did bother that they did not have a representation of their own body. We investigated their data and found that three of them already had experiences in VR. Based on the responses we received in the interviews, the reasons why they felt bothered vary from a gaming- 
background ("In most computer games you see at least your hand or arm.") to the mentioning of this fact as a general issue of VR. Despite this negative characterization, the participants did not characterize the overall experience negatively.

One major part we were analyzing in our data was the interaction between the immersed presenter and the virtual characters controlled from outside the VR. Our coding structure contained the dimensions verbal and non-verbal communication. The non-verbal dimension relates to the animations or movement of the virtual characters.

Participants in both groups rated animations similar. In our interviews we asked for a rating from 0 to 10 (where 0 is the lowest score) how natural the participants perceived the movements of the avatars in the virtual audience. As depicted in figure 6 , the median of the control group was 5.5 while the experimental group rated the interactions with a median of 6.5. The difference is not significant $(T=-1.266, d f=22, p=0.219)$.

In the interviews, participants characterized the movements as jerky or mechanic. However, most participants characterized it still as natural or partly natural.

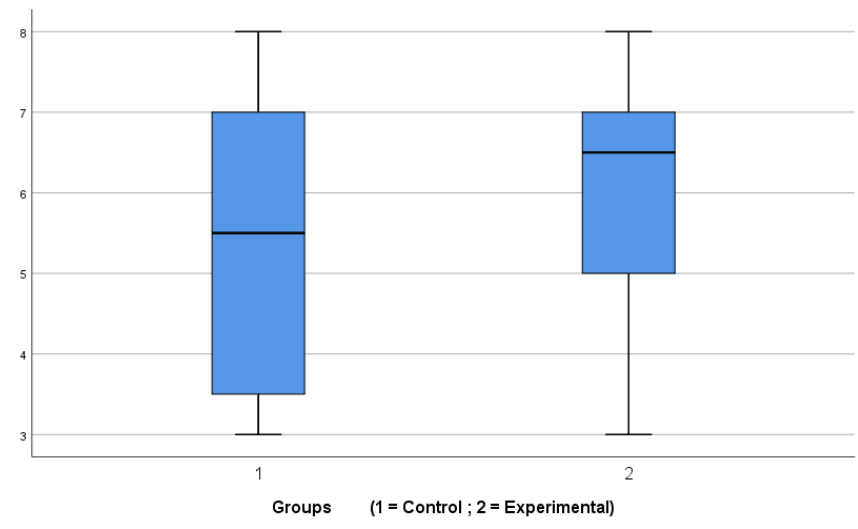

Fig. 6. Boxplot of scores of the scores of the avatar's movements sorted by the two groups.

We were particularly interested in the animation when an avatar raises the hand in order to request to speak. We had regular synthetic animations in the control group and the real-time movement of the orchestrator from outside the VR. Participants in the control group characterized the signaling as mostly natural: six rated it as natural, four were undecided or answered something in between (e.g. partly natural) and two perceived it as unnatural. The participant description of the movement indicated a more negative perception. Examples for characterizations from participants from this group were stiff, robotic or delayed. The participants who perceived the animations more natural gave characterizations such as harmonious or salient. We analyzed if we could find a relation to the score of the questionnaire assessing a tendency towards social phobia (Mini-SPIN), a score of at least 6 would indicate a tendency. Two participants that rated the animations as unnatural had both a very low score on the Mini-SPIN: one scoring 0, which is the lowest score possible, while the second scored 2. The other participants ranged between 3 and 5 which indicates no tendency towards social phobia.

The verbal interaction differed within the groups as well: the control group got pre-recorded statements while the experimental group got direct voice input from the orchestrator outside the VR. We scripted the audience's statements and questions during the design of the study. The orchestrator used in the experimental condition the same text as pre-recorded. The statements came at specific moments during the presentation, e.g. when the presenter mentioned a fact on a slide. We observed that in total 16 participants asked follow-up questions (control group: 8; experimental group: 8). The following example illustrates this:

Presenter [talking about places to visit in Italy]

Presenter [scripted]: Has anybody been to one of these places?

Avatar [scripted]: Yes, I have been in Rome and visited the Colosseum and Vatican.

Presenter: Oh, nice. Which of the places you liked more?

Based on the group the participant was assigned to, the avatar was able to follow up or not. For the control group we did not pre-record any responses to follow-up questions. The variety of possible questions made it almost impossible to anticipate all questions and pre-record the answers. Hence, the avatars remained silent and no answer was provided what may affect the pobic stimulus. In the experimental group, the orchestrator was able to react to the situation and respond spontaneously. The presenters got an answer from the avatars and continued the presentation after they were satisfied with the answer.

The lack of verbal interactivity in the control group was rated by seven participants during the interviews negatively. Most of them mentioned that they were wondering why the avatars did not answer and that they expected a different reaction. Our observations confirmed this. We observed that participants were hesitating and waiting before they resumed the presentation. One even started laughing and was asking the audience Hello? Anybody there?. We received feedback that this made the whole experience unnatural and was even characterized as break in presence.

On the other hand, the experimental group did not rate the possibility of interaction as highly positive. Six participants mentioned during their interviews that were assigned to the code "verbal interaction". They rated the interaction with the avatars positive and as one of the best experiences during the experiment. The only critique was towards the statements, the orchestrator gave during the experiment. One participant told us that they were too generic and it would be better to have more specific and personal statements included.

Another point of interest was the effect of a presentation training in VR. We asked the participants if they think that 
such a system would help them in their presentations. $80 \%$ of the participants mentioned that they would benefit after exercising presentations in this environment. They emphasized that the system must allow them to present their own presentation rather than a totally generic presentation. The participants agreed that a training only once would not help, the effect of training will persist when the training is done on a regular basis. The exercise is better perceived compared to a walktrough or presentation at home. One participant highlighted that the virtual environment looks very real and this would support the training effect.

Four participants did state that they would not benefit from this system. They all characterized themselves as good presenters with a high self-confidence. This is - except for one participant - supported by low scores on the Mnin-SPIN questionnaire which range between 0 and 2 . One participant feels that the training effect will not sustain as he/she feeling as being in "different worlds" when presenting in VR compared to real-life. This participant is scoring on the Mini-SPIN a score of 4.

Our observations revealed that most participants did not move much during the presentation. We observed that most were moving in a range of about two by three meters, although they knew that they were able to walk freely within the borders. One question in our interviews was dedicated to the walking and if the participants did see the chaperone-system (code: movement). Five (two in the experimental, three in the control group) stated that they walked to the borders and the chaperone system appeared. One participant explained that he/she wanted to assure that the system is working and the border is still there at the beginning of the presentation. One participant walked towards an avatar and insisted to close the notebook. While walking to the avatar, the chaperone system appeared and restricted the participant.

The use of controllers was in most cases unproblematic and intuitive for the participants. During our observations we noted only one participant who had troubles with using the controller. The troubles occurred when the participant wanted to point on the slides with the laser pointer but instead switched the slides. After this, no other issues were noticed or mentioned during the interview.

\section{DISCUSSION}

The presented study evaluated a system that allows an orchestrator of a social VR experience to interact directly with an immersed user by controlling an avatar's verbal and non-verbal interactions. The system is focusing on the usecase of presentations. In the study, each participant presented and, depending on the group, received direct interaction from the orchestrator outside the VR or pre-scripted interactions. All interactions contained a verbal and non-verbal dimension.

On the non-verbal dimension, we took over the avatar's behavior only in case of a direct interaction between the presenter and virtual character controlled by the orchestrator. When there was no direct interaction, both groups saw regular synthetic animations. This implies that the time, when the immersed presenter saw the orchestrator's live interaction, was short compared to the time of synthetic animations. The rational behind this design was that we cannot take-over all avatars because the immersed presenter would recognize when all avatars behave exactly same. Our implementation contained animations repeated by all avatars in random orders. If the orchestrator would control one avatar during the whole presentation, it would behave differently compared to all others in the virtual audience. Besides that, the transition to take over other avatars would not be easily possible or might cause a break in presence.

Of specific interest was, whether the take-over of an avatar is influencing the overall-experience of the non-verbal interaction. Our results indicate that the participants did not see a disruptive character. This finding opens up for further uses of such systems. In settings with a closer interaction than in presentations (e.g. in oral exams), we might use the tracking to deliver gesticulation as additional stimulus to verbal interaction.

The results addressing the verbal interaction, overall, indicate that participants had a better experience when the live interaction was enabled. However, we did not integrate voice modelling which allows the transformation of statements to a different voice, what may be a key in future uses. This may foster a better experience as the orchestrator can take the role of avatars of all genders. Furthermore, we might provide avatars having individual voices. We argue that this is essential for the use in real settings. Participants recognized the researcher's voice, which probably caused a break in presence ("I knew it was you, so I knew this is not real").

Our system made it necessary that the orchestrator is sitting in a different room. The reason is that the participants in the VR heard the voice double, once from the researcher and out of the headphones, even when using noise-cancelling headphones. On one hand, this opens up the opportunity to deliver therapy over distances using the internet as demonstrated in other systems [37]. On the other hand, we see the opportunity to use the system in other use-cases where social interaction plays a role, e.g. in the training of oral exams or job interviews.

Our results suggest that the system itself is creating a high sense of presence and social presence in particular. Our observations and coding of interviews point out that the participants felt as comfortable as in a real presentation scenario. Between the groups we did not detect any difference in terms of social presence and presentation comfort. This effect was expected because both groups performed the same presentation under the same influencing factors of presence [36].

As the social and co-presence did not differ in both groups significantly, we conclude that the integration of motion tracking during parts of the experiment did not compromise the perception of avatars. The take-over of avatars caused a different behavior and interaction compared to other avatars 
and before the take-over. As the questionnaire is assessing the behavior of virtual humans and the participant's perception of them, we expected that a possible negative impact may be identified by them.

Overall, results indicate that our system is able to bring benefit in terms of enriching therapist control when patients are exercising presentations compared to other systems using pre-recorded animations and audio clips to interact [19]. The systems enables therapists to deliver highly individualized phobic stimuli spontaneously. However, in order to deliver a good and purposeful therapy, the patients should be able to present their own slides and presentations. Our study design included prepared Power Point slides to ensure data quality, i.e. comparable presentation time between the participants and we needed pre-recorded audio clips for the interaction with the presenter.

Our results imply that therapists may benefit from the rich interactions implemented in our system. First, the system may provide a more flexible therapy as the therapist can act spontaneously. This leads to a greater flexibility and the opportunity to employ interactive role-plays. Second, in systems that do not allow spontaneous verbal interaction, therapists need to prepare every session individually which may involve prerecording of statements. In contrast, our system does not demand any pre-recorded interactions what may contribute to a more efficient therapy process.

\section{Limitations}

Our study contains limitations that we are aware of. In terms of user safety and lack of therapeutical accompaniment during data collection, we only recruited participants not diagnosed with any form of social phobia. As we are not exploring the efficiency of the prepared scenario as a treatment environment for phobias but investigate the possibility to create rich interactions with a therapist, we argue that this does not compromise the outcome of this study. A healthy participant can experience and evaluate the interactions created with our systems. It may be even possible that healthy participants are more suitable to evaluate our system as they are experiencing a lower emotional activation. Furthermore, the development process for mental health [1] contains two phases of which the first phase is lead by HCI researchers [2] and evaluated with healthy participants. We consider our system as part of this first phase as it is exploring the technological possibilities and evaluating the first implementation of a concept.

Moreover, the verbal interaction in the experimental group presents a limitation. In our experiment, the direct voice input stems from the researcher who was conducting the whole experiment including the introduction. This implies that the participants knew the voice in advance. Furthermore, the orchestrator could only choose characters of his gender as we did not implement any voice modulation. In our prerecorded condition, we also had statements that were recorded from a female speaker. The live-condition did not contain this possibility.

\section{CONClusion}

In conclusion, the use of such a system provides the orchestrator with more flexibility to conduct a training or therapy. Especially, the verbal interaction provided more flexibility, which fosters a better experience by the presenters. We did not find any indication of a negative impact that the takeover of animations of the avatars on the presenters had. This provides evidence that this method can also be used in future settings.

We see the benefit in further scenarios of social phobia that involve direct and closer interactions as for instance in a direct dialogue between an immersed user and a virtual character. Direct dialogues may be used in the treatment of different social anxieties, e.g. talking to a random person or in oral exams.

To come back to our inital hypothesis that the experimental group will experience a more natural verbal interaction we can conclude that our results support this hypothesis. The results from our qualitative analysis indicate that the participants are feeling more comfortable when they received a spontaneous answer. Our observations revealed that participants tend to pause their presentation for a response of the audience when no direct interaction was enabled. These circumstances could lead to a break in presence.

The second hypothesis saying that the take-over of avatars is not influencing the participants negatively is supported by our data, as well. The subjective ratings on the avatar's animations did not provide evidence of a different behavior after the avatars were taken over. The change of movements was not perceived negatively by the participants.

\section{FUTURE WORK}

The concepts evaluated with our system are exemplified in the use-case of fear of presentations. The concepts indicated its possible usefulness and we intend to extend and improve the system to use it in further settings.

For the therapeutic use of the system, the user interface of the control unit and the interaction itself has to be evaluated with regard to usability and fault tolerance. To do so, we need to evaluate this with the respective user group. As described above, the next step in the design process for technology in mental health is to engage with the therapists. The intuitiveness of the gestures, the orchestration itself and their expert assessment of the use in therapy will be part of this study.

We expect that voice modelling will impact the real use in therapy significantly due to the direct relation to the therapist using the system. Therefore, we intend to integrate this feature in advance to a evaluation with therapists.

The motion tracking systems we use - the Microsoft Kinect v2 - is discontinued and we experienced issues while tracking the user. In some cases, it lost the tracking for a short time which resulted in unnatural movements of the avatars controlled. Even though we were able to minimize this issue the lost of tracking could not be resolved completely. However, 
the participants did not report any issues related to this system behaviour. Still, we intend to use a different hardware setup for further evaluations.

\section{ACKNOWLEDGEMENTS}

This research project is funded by the German Federal Ministry of Education and Research under the Grant-Number 13GW0169.

\section{REFERENCES}

[1] D. Coyle, G. Doherty, M. Matthews, and J. Sharry, "Computers in talkbased mental health interventions," Interacting with Computers, vol. 19, no. 4, pp. 545-562, 2007.

[2] G. Doherty, D. Coyle, and M. Matthews, "Design and evaluation guidelines for mental health technologies," Interacting with Computers, vol. 22, no. 4, pp. 243-252, 72010.

[3] A. Stork, N. Sevilmis, D. Weber, D. Gorecky, C. Stahl, M. Loskyll, and F. Michel, "Enabling virtual assembly training in and beyond the automotive industry," in 2012 18th International Conference on Virtual Systems and Multimedia. IEEE, 9 2012, pp. 347-352.

[4] H. Liang, F. Liang, F. Wu, C. Wang, and J. Chang, "Development of a VR prototype for enhancing earthquake evacuee safety," in Proceedings of the 16th ACM SIGGRAPH International Conference on VirtualReality Continuum and its Applications in Industry - VRCAI '18. New York, New York, USA: ACM Press, 2018, pp. 1-8.

[5] K. Kim and J. Park, "Virtual bone drilling for dental implant surgery training," in Proceedings of the 16th ACM Symposium on Virtual Reality Software and Technology - VRST '09. New York, New York, USA: ACM Press, 2009, p. 91.

[6] B. O. Rothbaum, L. F. Hodges, R. Kooper, D. Opdyke, J. S. Williford, and M. North, "Virtual reality graded exposure in the treatment of acrophobia: A case report," Behavior Therapy, vol. 26, no. 3, pp. 547554, 221995

[7] H. U. Wittchen, F. Jacobi, J. Rehm, A. Gustavsson, M. Svensson, B. Jönsson, J. Olesen, C. Allgulander, J. Alonso, C. Faravelli, L. Fratiglioni, P. Jennum, R. Lieb, A. Maercker, J. van Os, M. Preisig, L. Salvador-Carulla, R. Simon, and H.-C. Steinhausen, "The size and burden of mental disorders and other disorders of the brain in Europe 2010," European Neuropsychopharmacology, vol. 21, no. 9, pp. 655679, 2011.

[8] R. C. Kessler, P. Berglund, O. Demler, R. Jin, K. R. Merikangas, and E. E. Walters, "Lifetime Prevalence and Age-of-Onset Distributions of DSM-IV Disorders in the National Comorbidity Survey Replication," Arch Gen Psychiatry, vol. 62, no. 6, pp. 593-602, 62005.

[9] Y. Choy, A. J. Fyer, and J. D. Lipsitz, "Treatment of specific phobia in adults," Clinical Psychology Review, vol. 27, no. 3, pp. 266-286, 2007.

[10] D. Opriş, S. Pintea, A. García-Palacios, C. Botella, . Szamosközi, and D. David, "Virtual reality exposure therapy in anxiety disorders," Depression and Anxiety, vol. 29, no. 2, pp. 85-93, 2012.

[11] C. Botella, J. Fernández-Álvarez, V. Guillén, A. García-Palacios, and R. Baños, "Recent Progress in Virtual Reality Exposure Therapy for Phobias: A Systematic Review," p. 42, 72017.

[12] P. M. G. Emmelkamp, M. Bruynzeel, L. Drost, and C. A. P. G. van der Mast, "Virtual Reality Treatment in Acrophobia: A Comparison with Exposure in Vivo," CyberPsychology \& Behavior, vol. 4, no. 3, pp. 335-339, 2001.

[13] A. Mühlberger, "Efficacy of a one-session virtua reality exposure treatment for fear of flying," Psychotherapy Research, vol. 13, no. 3, pp. 323-336, 2003.

[14] A. Mühlberger, A. Weik, P. Pauli, and G. Wiedemann, "One-session virtual reality exposure treatment for fear of flying," Psychotherapy Research, vol. 16, no. 1, pp. 26-40, 2006.

[15] M. B. Powers and P. M. Emmelkamp, "Virtual reality exposure therapy for anxiety disorders: A meta-analysis," Journal of Anxiety Disorders, vol. 22, no. 3, pp. 561-569, 42008.

[16] A. Garcia-Palacios, H. G. Hoffman, S. Kwong See, A. Tsai, and C. Botella, "Redefining Therapeutic Success with Virtual Reality Exposure Therapy," CyberPsychology \& Behavior, vol. 4, no. 3, pp. 341-348, 62001.

[17] A. P. Association et al., Diagnostic and statistical manual of mental disorders (DSM-5®). American Psychiatric Pub, 2013.
[18] S. Stupar-Rutenfrans, L. E. H. Ketelaars, and M. S. van Gisbergen, "Beat the Fear of Public Speaking: Mobile $360^{\circ}$ Video Virtual Reality Exposure Training in Home Environment Reduces Public Speaking Anxiety," Cyberpsychology, Behavior, and Social Networking, vol. 20, no. 10, pp. 624-633, 102017.

[19] D.-P. Pertaub, M. Slater, and C. Barker, "An Experiment on Public Speaking Anxiety in Response to Three Different Types of Virtual Audience," Presence: Teleoperators and Virtual Environments, vol. 11, no. 1, pp. 68-78, 22002.

[20] B. G. Witmer and M. J. Singer, "Measuring Presence in Virtual Environments: A Presence Questionnaire," Presence: Teleoperators and Virtual Environments, vol. 7, no. 3, pp. 225-240, 61998.

[21] M. Slater and S. Wilbur, "A framework for immersive virtual environments (five): Speculations on the role of presence in virtual environments," Presence: Teleoperators and Virtual Environments, vol. 6, no. 6 , pp. 603-616, 121997.

[22] Y. Ling, H. T. Nefs, N. Morina, I. Heynderickx, and W.-P. Brinkman, "A Meta-Analysis on the Relationship between Self-Reported Presence and Anxiety in Virtual Reality Exposure Therapy for Anxiety Disorders," PLoS ONE, vol. 9, no. 5, p. e96144, 52014.

[23] M. Slater, "Place illusion and plausibility can lead to realistic behaviour in immersive virtual environments," Philosophical Transactions of the Royal Society of London B: Biological Sciences, vol. 364, no. 1535, 2009.

[24] K. L. Nowak and F. Biocca, "The Effect of the Agency and Anthropomorphism on Users' Sense of Telepresence, Copresence, and Social Presence in Virtual Environments," Presence: Teleoperators and Virtual Environments, vol. 12, no. 5, pp. 481-494, 102003.

[25] C. Youngblut, "Experience of presence in virtual environments," INSTITUTE FOR DEFENSE ANALYSES ALEXANDRIA VA, Tech. Rep., 2003.

[26] S. Poeschl and N. Doering, "Measuring Co-Presence and Social Presence in Virtual Environments - Psychometric Construction of a German Scale for a Fear of Public Speaking Scenario." Studies in health technology and informatics, vol. 219, pp. 58-63, 2015.

[27] H. Trinh, R. Asadi, D. Edge, and T. Bickmore, "RoboCOP," Proceedings of the ACM on Interactive, Mobile, Wearable and Ubiquitous Technologies, vol. 1, no. 2, pp. 1-24, 62017.

[28] N. Kang, W.-P. Brinkman, M. Birna van Riemsdijk, and M. Neerincx, "The design of virtual audiences: Noticeable and recognizable behavioral styles," Computers in Human Behavior, vol. 55, pp. 680-694, 22016.

[29] M. Chollet, T. Wörtwein, L.-P. Morency, A. Shapiro, and S. Scherer, "Exploring feedback strategies to improve public speaking," in Proceedings of the 2015 ACM International Joint Conference on Pervasive and Ubiquitous Computing - UbiComp '15. New York, New York, USA: ACM Press, 2015, pp. 1143-1154.

[30] M. Chollet, K. Stefanov, H. Prendinger, and S. Scherer, "Public Speaking Training with a Multimodal Interactive Virtual Audience Framework," in Proceedings of the 2015 ACM on International Conference on Multimodal Interaction - ICMI '15. New York, New York, USA: ACM Press, 2015, pp. 367-368

[31] M. Chollet, P. Ghate, C. Neubauer, and S. Scherer, "Influence of Individual Differences when Training Public Speaking with Virtual Audiences," in Proceedings of the 18th International Conference on Intelligent Virtual Agents - IVA '18. New York, New York, USA: ACM Press, 2018, pp. 1-7.

[32] P. Lindner, A. Miloff, S. Fagernäs, J. Andersen, M. Sigeman, G. Andersson, T. Furmark, and P. Carlbring, "Therapist-led and self-led onesession virtual reality exposure therapy for public speaking anxiety with consumer hardware and software: A randomized controlled trial," Journal of Anxiety Disorders, 72018.

[33] M. Koller, P. Schäfer, M. Sich, J. Diemer, M. Müller, and G. Meixner, "Next Generation Virtual Reality Exposure Therapy Systems - A Study exploring Design Implications," in 2018 International Conference on Intelligent Systems (IS). IEEE, 2018.

[34] K. M. Connor, K. A. Kobak, L. E. Churchill, D. Katzelnick, and J. R. Davidson, "Mini-SPIN: A brief screening assessment for generalized social anxiety disorder." Depression and anxiety, vol. 14, no. 2, pp. 137-40, 2001

[35] J. Wiltink, S. Kliem, M. Michal, C. Subic-Wrana, I. Reiner, M. E. Beutel, E. Brähler, and R. Zwerenz, "Mini - social phobia inventory (mini-SPIN): psychometric properties and population based norms of the German version." BMC psychiatry, vol. 17, no. 1, p. 377, 2017. 
[36] M. V. Sanchez-Vives and M. Slater, "From presence to consciousness through virtual reality," Nature Reviews Neuroscience, vol. 6, no. 4, pp. 332-339, 42005.

[37] D. Hartanto, W.-P. Brinkman, I. L. Kampmann, N. Morina, P. G. M. Emmelkamp, and M. A. Neerincx, "Home-Based Virtual Reality Exposure Therapy with Virtual Health Agent Support," 2016, pp. 85-98. 